\title{
Introducción: Variedad de temas y de tratamientos sobre la Innovación
}

\section{Introduction: A Variety of Innovation Topics and Approaches}

\author{
Mikel Gómez Uranga ${ }^{1}$ \\ Goio EtXebarria Kerexeta ${ }^{2}$ \\ Universidad del País Vasco (UPV/EHU) (España)
}

$\mathrm{N}^{\circ}$ de clasificación JEL: O30, O31, O39

DOI: $10.5295 / \mathrm{cdg} .120329 \mathrm{mg}$

\section{Resumen:}

Este artículo de introducción al número especial sobre innovación de la revista Cuadernos de Gestión consta de dos apartados bien diferenciados. En el primero, se recogen algunas ideas que pueden ser útiles para los lectores que se aproximan al tema de la innovación y observan una gran variedad conceptual. En concreto, se ha tratado de explicar de una manera más o menos exhaustiva el concepto de Open Innovation (OI). La OI surge del reconocimiento de que la empresa no puede innovar de manera aislada, y, por lo tanto, necesita siempre adquirir las ideas y los recursos del entorno exterior. Por otro lado, el concepto de Sistemas Regionales de Innovación (RIS), ampliamente tratado en la literatura sobre innovación y desarrollo regional en los últimos quince años, aporta algunas ideas que pueden ser complementarias con la OI. Los autores van a caracterizar y diferenciar ambos conceptos. En una segunda parte del artículo, se presentan e introducen brevemente el resto de los artículos que componen este número monográfico sobre innovación.

Palabras clave:

Innovación abierta, Sistemas Regionales de Innovación, networking.

\begin{abstract}
:
This introductory article to the special issue of the journal Cuadernos de Gestion consists of two clearly differentiated sections. The first contains some ideas which may prove useful for readers approaching the topic of innovation and who find a wide range of concepts. Special care has been taken to offer a full explanation of the concept of "Open Innovation" (OI). OI arises from companies having realized that they cannot innovate when they are isolated. They constantly need to acquire ideas and resources from their external environment. As regards the concept of Regional Innovation Systems (RIS), which have been widely discussed in literature on innovation and regional development during the last fifteen years, some ideas which may be complimentary to OI are presented. The authors go on to show the characteristics and differences between the two concepts. The second section gives a brief overview of the rest of the articles that make up this monographic issue on innovation.
\end{abstract}

Keywords:

Open Innovation, Regional Innovation Systems, networking.

\footnotetext{
${ }^{1}$ Departamento de Economía Aplicada I.Facultad de Ciencias Económicas y Empresariales - UPV/EHU. Avda. Lehendakari Agirre, 8348015 Bilbao. miguel.gomez@ehu.es

2 Departamento de Economía Aplicada I. Facultad de Ciencias Sociales y de la Comunicación - UPV/EHU. Barrio Sarriena s/n 48940 Leioa - Bizkaia. goio.etxebarria@ehu.es
} 


\section{INTRODUCCIÓN DE LA OPEN INNOVATION ENTRE OTROS CONCEPTOS DE INNOVACIÓN}

En este número especial de la revista Cuadernos de Gestión se presenta casi una fotografía actual de la variedad de tratamientos y de contenidos que se pueden encontrar en el tema de la innovación. Antes de presentar cada uno de los artículos que aparecen en este número de la revista, hemos querido introducir, como muestra también de la variedad, algunas ideas útiles para los lectores sobre la diversidad conceptual de la innovación a partir de una explicación, más o menos exhaustiva, del concepto de Open Innovation (OI).

En la actualidad existe una variedad importante de aproximaciones metodológicas para estudiar la innovación. Hace ya más de quince años que se consolidaron los conceptos de Sistemas de Innovación en sus dos dimensiones: Nacional (Nelson y Rosenberg, 1993) y Regional (Regional Innovation Systems, en adelante RIS) (Cooke et al., 1997). Estos conceptos respondían a una aproximación de carácter más holística. Casi al mismo tiempo se difundió el paradigma sobre clusters, que más tarde llegaría casi a confluir, o al menos a buscar un acercamiento, con el de RIS (Porter, 2003; Navarro, 2009).

En los últimos años se difunde una "Nueva geografía económica de la innovación" (Asheim et al., 2011), que hace hincapié en el uso de ciertas bases evolucionistas de corte clásico. En este siglo adquieren mayor relevancia aproximaciones más focalizadas en las relaciones de diversos agentes. Se trata de la "Triple Hélice" (Etzkowitz y Leydesdorff, 1996) donde interactúan tres tipos de agentes, y finalmente la Open Innovation (OI), que es precisamente la que tratamos de manera más exhaustiva en estas páginas. La elección obedece a que el objeto de análisis se encuentra vinculado a la gestión empresarial de la innovación, y por eso nos ha parecido la aproximación sobre la innovación más idónea para ser tratada en una revista como Cuadernos de Gestión.

Uno de los conceptos vinculado a la innovación más difundido en el mundo en los últimos años es el de Open Innovation. Existe una cantidad importante de artículos en las revistas de mayor prestigio. Las referencias más citadas de autores son los libros de Von Hippel (1988) y Chesbrough (2003), donde se encontrarían los orígenes del concepto.

En la coyuntura histórica en la que comenzaron los trabajos sobre la "gestión de la innovación", el mundo era más cerrado y los centros productivos se encontraban más concentrados en determinados lugares, y con ciertos niveles de autosuficiencia. Hoy, sin embargo, nos encontramos en un mundo más abierto y modular (Lakhani y Tushman, 2012). Un modelo estándar de Open Innovation (OI), está bien recogido en la siguiente definición: "La OI se ocupa de cómo las empresas comercializan sus invenciones y tecnologías a través de la venta o del licenciamiento de recursos desarrollados en otras organizaciones" (Dahlander y Gann, 2010, p. 704). Tanto los estudios seminales como los más actuales de la OI se han realizado sobre la open source software (Lakhani y Tushman, 2012). En este último caso se trataría de distinguir entre software cerrado, o propietario, y abierto, es decir que no excluya a otros grupos o personas a participar, como es el caso de IBM en 1981 (Huizingh, 2011; Lakhani y Tushman, 2012).

La OI surge del reconocimiento de que la empresa no puede innovar de manera aislada, $\mathrm{y}$, por lo tanto, necesita siempre adquirir las ideas y los recursos del entorno exterior. La 
OI contempla dos perspectivas diferentes: dentro de los límites de la empresa (inbound) o fuera (outbound). En el primer caso se trata de un uso interno del conocimiento adquirido sobre todo en el exterior; en el segundo, se trata de un uso externo por parte de la empresa del conocimiento interno (Huizingh, 2011). Para la empresa, ya se trate de conocimientos adquiridos internamente como externamente, las dos fuentes serían más complementarias que sustitutivas (Dahlander y Gann, 2010).

En los estudios sobre la OI se analizan las prácticas empresariales a través de ciertos criterios, como por ejemplo: inbound/outbound y pecunary/no pecunary. Las formas de "apertura" de las empresas son distintas según las propias circunstancias, que pueden ser evaluadas a partir de diferentes prácticas, valores y resultados. Por eso, Chesbourg define la OI como el conjunto de prácticas para mejorar la innovación, para lo cual desarrolla un modelo cognitivo para interpretar y buscar aquellas prácticas (Chesbourg, 2006; Dahlander y Gann, 2010).

En los estudios sobre OI casi siempre se da por supuesto que la "apertura" reporta a las empresas unos beneficios o unas determinadas ventajas, que es interesante poder evaluar. Pero ya es más difícil encontrar investigaciones que hagan hincapié en las desventajas o en los costes de la apertura. No hay modelos interpretativos asentados y consensuados internacionalmente que se aproximen a esa tarea. Una parte relevante de los autores que tratan las ventajas/desventajas de la OI, se fundamentan en los llamados neoinstitucionalistas norteamericanos, que utilizan diversas categorías analíticas como: costes de transacción, toma de decisiones entre mercado/empresa a la hora de realizar ciertas transacciones e intercambios. Nos referimos a Coase (1937) y Williamson (1975).

Algunos autores adjudican variables específicas para una medición aproximada de los costes/beneficios de la OI, como por ejemplo: número de innovaciones alcanzadas y beneficios financieros generados (Huizingh, 2011), o un estudio de las "barreras de adopción" de la OI (Mortara y Minshall, 2011). En todo caso, parece un camino en el que es necesario avanzar en los próximos años.

En la literatura sobre la OI encontramos estudios tanto sobre industrias como sobre determinadas empresas. Donde más estudios se realizan es en el área de las grandes multinacionales pero sobre todo en sectores muy intensivos en tecnología, tanto en innovaciones radicales como de producto o de proceso. Destacan estudios de empresas emblemáticas y líderes como por ejemplo: Apple y Philips (Lakhani y Tushman, 2012; Huizingh, 2011; Todtling et al., 2011). Otro estudio indaga sobre los diferentes caminos de la OI en 43 grandes multinacionales (Mortara y Minshall, 2011). Aunque donde más estudios se realizan es en áreas de grandes multinacionales, sin embargo también se encuentran algunos sobre Pymes, como el artículo de Lee et al. (2010). Esos autores consideran que para las grandes empresas la OI se encuentra más limitada a las fases de cabecera, como son I+D e Invenciones; mientras que para las Pymes, que son las que estudian para el caso de Corea, la OI se encuentra más vinculada a otros actores en el downstream y marketing. Esto también se puede ver en el cuadro siguiente, donde se representan redes de colaboraciones. 
Figura 1

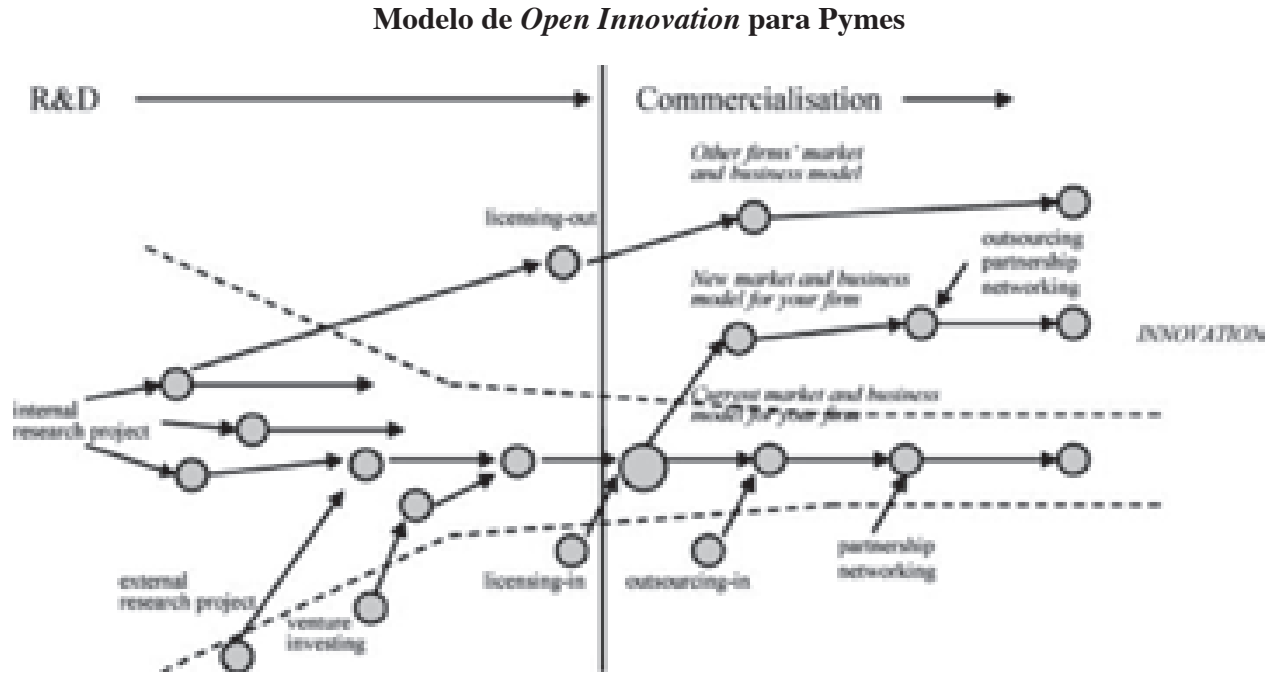

Fuente: Lee et al. (2010, p. 292)

La implementación de la OI es consustancial al funcionamiento de las redes. Las empresas tienen sus colaboradores primordiales para la OI, en proveedores, clientes, etc. En las Pymes se trataría de colaborar para la innovación con empresas especializadas, por ejemplo en marketing. En el análisis de colaboración en red, adquiere un papel importante el marco de "intermediarios" que permite poner en contacto a los colaboradores (Lee et al., 2010).

La implementación de las tecnologías más avanzadas en la actualidad favorece, y en ocasiones hace posible, el buen funcionamiento y la gestión de las redes de innovación. Nos parece más importante destacar que la OI, en el marco de la empresa, supone en muchos casos la implantación de "nuevos modelos de negocios". En efecto, se trataría de ir más allá que la mera adopción de nuevas prácticas. En esa dirección, Huizingh (2011) propone un modelo para implantar la OI, en el que se contemplan, entre otras, las siguientes actividades: búsqueda de oportunidades, evaluar mercados potenciales, crear mecanismos efectivos de gobernanza, y equilibrar incentivos y controles. En todo caso, en todos los modelos de OI analizados se destaca la importancia de la alta cualificación y de los skills más convenientes para los gestores y los empleados empresariales implicados en la innovación (Lakhani y Tushman, 2012).

En la generación de un marco empresarial de innovación influyen, tanto la capacidad de la empresa para absorber una parte del conocimiento e ideas que existen en el seno de la propia empresa, como elementos de contexto como las necesidades de innovación y de los diversos agentes que se sitúan en la red, y la cultura organizacional. En definitiva se trata de mejorar las capacidades de absorción de las empresas para poder enfrentarse en mejores condiciones a la OI (Rodríguez-Castellanos et al., 2010). A estos elementos de contexto se 
les pueden añadir otros más amplios, como aspectos socioculturales que tienen influencia de las redes. De ahí se puede deducir que la base territorial (en muchos casos regional) en la que descansan las redes, por ejemplo la capacidad de absorción de conocimiento de la región, influye en la mejora de los caminos de la OI.

En ese marco de base territorial las universidades de excelencia son centros a los que acuden las empresas y que fundamentan bases colaborativas importantes de transferir conocimientos, y donde la regulación de acuerdos sobre propiedad intelectual (licencias y patentes) son cruciales.

Todo lo anterior, al ser ya tratado en otros paradigmas metodológicos sobre la innovación, les lleva a algunos autores a sostener que el concepto de la OI no es nuevo. Las siguientes líneas muestran como ya en el siglo anterior en los años que se estaba concibiendo el concepto de RIS, algunos autores que estábamos en esa tarea, escribíamos algunas ideas que podían hoy ser complementarias con la OI: "Aunque en estas páginas concebimos la innovación como un resultado de las relaciones de carácter sistémico, sin embargo los resultados de lo que se entiende convencionalmente como innovación provienen de las relaciones de cooperación que se establecen en el núcleo del sistema productivo (en parte regional) entre empresas, proveedores y clientes. En ese núcleo se observan reacciones permanentes de carácter interactivo, así como efectos de aprendizaje. Un porcentaje mucho menor de la innovación procede, en cambio, de las transacciones originadas con las comunidades de universidades e institutos de investigación (en una parte públicos). Sin embargo, el núcleo del sistema productivo se encuentra inmerso en un determinado contexto local con el que permanentemente interactúa, es decir, que desde una perspectiva asistémica no se puede entender la generación de procesos innovadores, por lo que es necesario considerar las relaciones entre un conjunto de subsistemas que, en parte, se caracterizan por su proximidad territorial. (...) Una primera aproximación de "cultura productiva" haría referencia a ciertas representaciones de la base cultural vinculadas a la esfera productiva y a las áreas que con aquélla se conectan. Las relaciones entre el sistema productivo (cuyo núcleo principal está constituido por las empresas) y el sistema social determina el tipo de desarrollo de la región" (Cooke y Gómez Uranga, 1998, p. 58-59).

También el paradigma de cluster, muy utilizado en temas relacionados con innovación y política de innovación, para ser operativo, requiere que sea tenido en cuenta el contexto institucional (Lee et al., 2009).

A partir de esas bases, Todtling et al. (2011) desarrollan o conciben un nuevo término que denominan Regional Open Innovation, donde se fusionan la OI y el RIS. Aunque entendemos que se pueden utilizar diferentes caminos metodológicos, nos parece que desde un punto de vista epistemológico no es posible identificar los dos conceptos señalados. Metodológicamente las dos aproximaciones son muy diferentes y tienen centros de atención muy distintos. En el siguiente cuadro confrontamos algunas de las características de las dos áreas conceptuales. 
Tabla 2

Características de los RIS (Regional Innovation Systems) y de la OI (Open Innovation)

\begin{tabular}{|c|c|c|}
\hline CARACTERÍSTICAS & RIS & OI \\
\hline Foco de atención & $\begin{array}{l}\text { Interacción entre agentes del } \\
\text { sistema en el área regional }\end{array}$ & $\begin{array}{l}\text { Modos de "prácticas" en la } \\
\text { colaboración de las empresas con } \\
\text { otras en la innovación }\end{array}$ \\
\hline Output a conseguir & $\begin{array}{l}\text { Aumento de la Innovación } \\
\text { total en el área }\end{array}$ & $\begin{array}{l}\text { Mejora de los resultados de la } \\
\text { colaboración para innovar }\end{array}$ \\
\hline Agentes que intervienen & $\begin{array}{l}\text { Todos los que integran el } \\
\text { sistema }\end{array}$ & $\begin{array}{l}\text { Empresas implicadas con la OI, } \\
\text { intermediarios, y aquellas que } \\
\text { absorben el conocimiento }\end{array}$ \\
\hline Papel de la empresa & $\begin{array}{l}\text { Importante, pero debe } \\
\text { compartir con otros agentes }\end{array}$ & $\begin{array}{l}\text { Objeto principal de estudio } \\
\text { son sus comportamientos y sus } \\
\text { resultados }\end{array}$ \\
\hline $\begin{array}{l}\text { Tipos de mediciones } \\
\text { habituales }\end{array}$ & $\begin{array}{l}\text { Agregadas para el conjunto } \\
\text { de los agentes }\end{array}$ & $\begin{array}{l}\text { Medidas directas relacionadas con } \\
\text { la OI sobre: Patentes y licencias, } \\
\text { capacidad de absorción, y } \\
\text { resultados de la colaboración }\end{array}$ \\
\hline $\begin{array}{l}\text { Elementos que se } \\
\text { incorporan para la relación } \\
\text { inter-agentes }\end{array}$ & $\begin{array}{l}\text { Interfaces ad hoc, tanto } \\
\text { públicos como privados, para } \\
\text { generar colaboración }\end{array}$ & Intermediarios \\
\hline $\begin{array}{l}\text { Bases institucionales } \\
\text { formales }\end{array}$ & Leyes, regulaciones, normas & $\begin{array}{l}\text { Leyes de patentes y otras leyes de } \\
\text { ámbito empresarial }\end{array}$ \\
\hline $\begin{array}{l}\text { Clases de agentes del } \\
\text { sistema }\end{array}$ & $\begin{array}{l}\text { Indistintos: privados, } \\
\text { públicos o mixtos }\end{array}$ & $\begin{array}{l}\text { Preferentemente empresas } \\
\text { privadas }\end{array}$ \\
\hline $\begin{array}{l}\text { Agenda pendiente de } \\
\text { investigación }\end{array}$ & $\begin{array}{l}\text { Modelos teórico-empíricos } \\
\text { de relaciones entre RIS y lo } \\
\text { Global (marcos de análisis) }\end{array}$ & $\begin{array}{l}\text { Modelos acabados coste/beneficio } \\
\text { de la OI }\end{array}$ \\
\hline
\end{tabular}

Una de las conclusiones finales de esta primera parte es que los diversos paradigmas que se están utilizando entre los especialistas para aprehender el tema de la innovación son parciales, y se focalizan en problemas distintos. Pero pueden ser complementarios cuando se trata de encontrar explicaciones generales para el tratamiento teórico-empírico del fenómeno de la innovación.

\section{ARTÍCULOS QUE APARECEN EN ESTE NÚMERO ESPECIAL SOBRE INNOVACIÓN}

En el artículo "Las estrategias de especialización inteligente: Una estrategia territorial para las regiones", los autores M. Navarro, M.J. Aranguren y E. Magro realizan una 
revisión de la literatura actual sobre "estrategias regionales de innovación", que conduce a los mismos a conjeturar sobre "estrategias de especialización inteligentes".

En primer lugar, los autores distinguen entre "estrategias empresariales" y "estrategias territoriales", apartado este último en el que centran su atención. El territorio inicialmente se prefigura económicamente a través de su capacidad de atracción precisamente por el desarrollo de sus activos específicos. Un territorio avanzado compite por su disposición para innovar, aprovechando también las capacidades en las áreas científico-técnicas. Los autores consideran un avance importante en el tratamiento del territorio las contribuciones de los geógrafos económicos evolucionistas sobre la diversificación; esta aproximación se plasma en el concepto de related variety. Los autores entienden la learning region como una realidad asociada al concepto de "innovación incremental". La innovating region constituye un nivel más avanzado y completo que las regiones que aprenden, ya que se fundamenta en una base investigadora de mayor importancia.

La necesidad de "extroversión" de las regiones conduce a éstas a introducirse en redes de producción globales generadas por grupos multinacionales. Por lo tanto, las dinámicas innovadoras territoriales no pueden separarse fácilmente de los caminos trazados por grandes grupos empresariales globales.

Los autores se ocupan también de las "estrategias emergentes", donde participan múltiples agentes que concilian y coordinan sus intereses. A partir de ahí, se introducen en el área de la "gobernanza regional", sopesando los elementos que se asocian a la intervención de los gobiernos. En este punto encuentran diferencias con la visión de D. Foray, autor que construye el concepto de "especialización inteligente" que recoge la Comisión Europea; en su visión se trata de concentrar los recursos de $\mathrm{I}+\mathrm{D}+\mathrm{i}$ en unos pocos territorios más competitivos relativamente. Los autores analizan en el artículo la aproximación de Foray desde diversas perspectivas como son: la variedad, las políticas orientadas, y el posicionamiento de regiones más desarrolladas respecto a las tecnologías de uso genérico. Para Foray estas últimas tecnologías se desarrollarían exclusivamente en regiones avanzadas, y en cambio para regiones menos competitivas se reservaría, un conjunto de "invenciones y aplicaciones" menos innovadoras. Esta división del trabajo se acompaña con ejemplos como el de las nanotecnologías aplicadas en la alimentación. Y en otros campos aplicados que se relacionan con las bios. Los autores discrepan matizadamente con esa concepción, que tampoco compartimos los que escribimos esta introducción, ya que nos parece que esa división del trabajo únicamente sería aplicable en espacios muy dependientes, que no aspirarían a tener una capacidad de investigación y en consecuencia masa crítica de generación de conocimiento propia.

El artículo de M.A. Galindo, D. Ribeiro y M.T. Méndez, titulado "Innovación y crecimiento económico: Factores que estimulan la innovación”, parte de una lectura de Schumpeter que coloca al "empresario emprendedor" en un lugar central de la innovación. Ésta también depende, entre otros factores, del "clima social", en el que tienen una influencia directa la distribución de la renta y la formación. Con esos mimbres y apoyándose en el European Innovation Scoreboard de la Comisión Europea, los autores realizan un estudio empírico para nueve países europeos, además de EEUU y Japón.

El estudio empírico realizado en el artículo confirma que la innovación tiene una influencia directa en el crecimiento económico. Además, demuestran que tanto una política impositiva de redistribución de la renta, así como una política monetaria que aporte recur- 
sos para financiar la innovación, constituyen variables significativas para la generación de procesos de innovación. Este estudio tiene cierta dosis de originalidad, principalmente en la inclusión de variables como el porcentaje de emprendedores sobre el total de la población adulta del país, y el total de estudiantes universitarios del país.

En el artículo "Innovación y Política Tecnológica: el caso del Sector Transformador y el Sector Servicios a Empresas”, María Soledad Castaño analiza distintos modos de estudiar la innovación y qué factores son determinantes en el éxito de las actividades de I+D+i. Para ello realiza una revisión de los estudios que existen, tanto desde la perspectiva de los inputs de I+D (gastos de I+D y personal empleado en actividades de I+D) como del output (resultados de la innovación, en concreto, patentes). También recoge las aportaciones de autores, como Lawson y Samson, que sostienen que los efectos del capital humano y el conocimiento tecnológico acumulado como recursos de la empresa son determinantes de la innovación, aunque no suficientes para determinar la misma.

Por otra parte, la aparición de la política tecnológica es consecuencia de un fallo de mercado, en el sentido señalado por Arrow. Así, recoge una serie de medidas con las que cuenta el decisor político: para incentivar la inversión en I+D+i, para potenciar la difusión y la adquisición de los conocimientos científicos y técnicos, y para fomentar un entorno favorable para la innovación de las empresas. La autora analiza cómo las medidas de política tecnológica pueden o no ser eficientes.

En el estudio empírico de los determinantes de innovación de producto en los dos sectores que la autora analiza, el sector transformador y el sector servicios a empresas, se contempla un emprendedor schumpeteriano, que juega un papel esencial en la innovación. Tomando datos de 25 países, y analizando como factores el capital humano, la tecnología, el grado de competencia del mercado, las expectativas y el grado de internacionalización, concluye que no se observan grandes diferencias entre los dos sectores excepto en el caso del capital humano de los emprendedores, cuyo efecto es mayor en el sector transformador.

El artículo que tiene por título "Key business factors of eco-innovation: an integrative assesment from empirical studies review", de A. Pereira y X. Vence, realiza una amplia revisión de la literatura sobre eco-innovación (EI) en los últimos años. El artículo puede resultar extraordinariamente útil para aquellas personas que se quieran acercar a la investigación señalada. Pero también puede ser de interés para un lector que quiera simplemente conocer los artículos más relevantes sobre el tema en la esfera internacional, como se muestra en los cuadros apéndices ordenando cada artículo a modo de "cuaderno de notas", a través de los objetivos perseguidos y de las variables principales sobre las que trabajan, así como de los resultados principales obtenidos de cada estudio.

Para la UE la eco-innovación oscila entre lo que se entiende habitualmente como innovación medioambiental y la innovación propiamente dicha; la primera tiene unos efectos positivos aunque no comerciales, y la última se asocia a la comercialización. Sería introducirnos en una dialéctica que se movería entre bien público y esfera privada. El concepto de EI tiene un perfil de dedicación de recursos hacia negocios medioambientales. El concepto de EI responde a una lógica de aproximación de una teoría evolucionista, interaccionan sistemas ecológicos, institucionales y sociales.

Los autores tratan de encontrar aquellos elementos que consideran centrales para la eco- innovación. Analizan, entre otras cosas, de qué manera los siguientes elementos tienen mayor o menor influencia en la implementación de la EI: a) la dimensión empresarial; b) el 
tipo de sector industrial de que se trate, por ejemplo empresas más intensivas en energía, o más o menos polucionadoras; c) la historia de la empresa y el tipo de empresariado (puede ser el ejemplo schumpeteriano de empresario innovador); d) el ahorro de costes.

Finalmente, los autores también analizan lógicas asociadas al tirón de la demanda esperada (demand pull) como factor inductor primero de la EI. Aunque los autores no hacen hincapié en este punto, sí parece un ejemplo muy relevante la producción del vehículo eléctrico.

El artículo "Technology Outlook as a tool for the management of innovation" de Jon Mikel Zabala presenta una visión teórica sobre la gestión de la innovación y las herramientas para implementar la misma, así como una aproximación práctica dirigida a las Pymes al objeto de facilitarles herramientas como puede ser la vigilancia tecnológica.

La gestión de la innovación ha sido ampliamente estudiada en las últimas décadas por numerosos autores, desde múltiples disciplinas: ciencia, ingeniería, economía, gestión estratégica, sociología y psicología. Debe entenderse como un proceso central de la organización de las empresas, cuyo objeto es crear o explotar nuevas oportunidades. Para ello se requieren tres niveles en los procesos de gestión de la innovación: el estratégico, donde se toman las decisiones sobre la cultura, los valores y los objetivos de la empresa; el operativo, que dota a las firmas de las rutinas que posibilitan la consecución de los objetivos estratégicos; y el instrumental, que provee del conjunto de herramientas que permiten a las compañías convertir esas rutinas en práctica.

De entre las múltiples herramientas de gestión de la innovación, el autor se interesa por el uso de la Vigilancia Tecnológica por parte de las Pymes. Realiza un pormenorizado análisis de los tipos de información requerida, los procedimientos en la toma de decisiones empresariales, y las fases del proceso de vigilancia tecnológica: observar, analizar y usar. También estudia los roles que se corresponden con dichas etapas: observadores, analistas y decisores. Sin embargo, en la práctica las herramientas de gestión de la innovación, y en concreto la vigilancia tecnológica, se ven infrautiliazadas por las Pymes, debido a los costes y las competencias que se requieren para su correcta utilización.

El autor concluye recordando que la vigilancia tecnológica es un proceso participativo que requiere un fuerte compromiso y una comunicación continua y coordinada. Las empresas necesitan tiempo para establecer un proceso de vigilancia tecnológica, que va a implicar cambios en sus rutinas organizativas. Asimismo, las herramientas de gestión han de adaptarse a las características de cada firma y a su ámbito local.

Hay que subrayar el interés práctico y la utilidad que este artículo puede proporcionar tanto a las Pymes como a los decisores políticos a la hora de diseñar sus estrategias de innovación.

En el artículo "La gestión de la innovación como la gestión de un eco sistema heterogéneo y estructurado", J.F. Arango presenta algunas características de una aproximación evolucionista a la innovación. Esta última se inicia con una propuesta creativa como es que los elementos sociales integran también a la innovación. Una novedad adquiere valor al relacionarla con lo existente, lo que crea tensiones, aunque hay contextos donde las innovaciones son mejor aceptadas.

La innovación opera en ecosistemas heterogéneos que contienen: precios, características, individuos, así como también intereses y valores. Habrá que gestionar las interacciones entre los elementos del ecosistema, y acoplar nuevos dispositivos tecnológicos e 
institucionales. El autor realiza una original elección sobre el estudio de caso. Se trata de un ecosistema de las "edificaciones sismo-resistentes", las innovaciones que se pueden introducir en un marco de edificaciones no preparadas para los movimientos sísmicos en Colombia. Los dispositivos sismo resistentes se encuentran en el marco de reglamentos, leyes y regulaciones específicas.

En el artículo "La arqueología comercial en España: ¿Un sistema sectorial de información?”, E. Parga, E. Castro e I. Fernández de Lucio se sitúan metodológicamente en la aproximación tradicional de Sistemas de Innovación, y específicamente de "sistemas sectoriales de innovación y de producción" de Malerba. Escogen un sector muy original en los tratamientos sobre innovación. Se trata de la arqueología desde la perspectiva comercial. Se observan conjuntos diversos de empresas que se relacionan con el sector estudiado, y aparece el sector de la construcción como tractor de todos esos negocios vinculados a la arqueología. Debido a la crisis tan intensa que sufre aquel sector en España, las empresas relacionadas con la arqueología se han visto notablemente reducidas.

El trabajo de investigación se orienta en reconocer cómo aprenden e innovan los “agentes" que integran el sector. Para el tratamiento técnico de base utilizan un sistema de encuestas y entrevistas previas a un panel de expertos. Entre las diferentes áreas o bloques de preguntas, nos interesa especialmente el que se refiere a la actividad innovadora de las empresas. Un capítulo de gran interés derivado del trabajo realizado, es el capítulo de las interacciones de los agentes. Dicho en otros términos, con quién y cómo colaboran los diferentes agentes, que como se sabe es la propiedad cualitativa más relevante a la hora de estudiar un sistema de innovación. Los resultados obtenidos son los siguientes: Las empresas de arqueología colaboran (o co-operan) en: proyectos, publicaciones, incorporación de bienes de equipo, formación de recursos humanos; con otras empresas de gestión arqueológicas, proveedores, comunidad científica y redes profesionales.

\section{REFERENCIAS BIBLIOGRÁFICAS}

ASHEIM, B.T.; BOSCHMA, R. y COOKE, P. (2011): “Constructing Regional Advantage: Platform Policies Based on Related Variety and Differentiated Knowledge Bases", Regional Studies, 45:7, 893-904.

CHESBROUGH, H.W. (2003): Open Innovation: The New Imperative for Creating and Profiting from Technology. Harvard Business School Press, Boston.

CHESBROUGH, H.W. (2006): "Open innovation: A new paradigm for understanding industrial innovation". En H.W. Chesbrough, W. Vanhaverbeke y J. West (Eds.): Open Innovation: Reaching a New Paradigm. Oxford University Press, New York, 1-12.

COASE, R. (1937): "The Nature of the Firm". Economica (Blackwell Publishing) 4 (16): 386-405.

COOKE, P. y GOMEZ URANGA, M. (1998): "Dimensiones de un sistema de innovación regional: organizaciones e instituciones", Ekonomiaz, No 41, pp. 46-67.

COOKE, P., URANGA, M.G. y ETXEBARRIA, G. (1997): "Regional systems of innovation: Institutional and organisational dimensions", Research Policy, 26, 475-491.

DAHLANDER, L. y GANN, D.M. (2010): "How open is innovation?" Research Policy, Vol. 39, N 6, 699-709.

ETZKOWITZ, H. y LEYDESDORFF, L. (1996): "Emergence of a Triple Helix of University- Industry-Government Relations", Science and Public Policy, Vol. 23, pp. 279-286. 
HUIZINGH, E.K.R.E. (2011): "Open innovation: state of the art and future perspectives". Technovation, Vol. 31, No 1, 2-9.

LAKHANI, K.R. y TUSHMAN, M.L. (2012): "Open innovation and organizational boundaries: The impact of task decomposition and knowledge distribution on the locus of innovation". Working Paper 12-057. Harvard Business School.

LEE, S.; PARK, G.; YOON, B. y PARK, J. (2010): “Open innovation in SMEs-an intermediated network model". Research Policy, 39, 290-300.

LEE, Y-S.; TEE, Y-C. y KIM, D-W. (2009): "Endogenous ver exogenous development: A comparative study of biotechnology industry cluster policies in South Korea and Singapore", Environment and Planning C. Government and Policy, 27, 612-631.

MORTARA, L. y MINSHALL, T. ( 2011): "How do large multinational companies implement implement Open Innovation?", Technovation, 31, 586-597.

NAVARRO, M. (2009): "Los sistemas regional de innovación. Una revisión crítica”, Ekonomiaz, N 70, pp. 24-59.

NELSON, R.R. y ROSENBERG, N. (1993): "Technical innovation and national systems", en OECD (2001), Devolution and Globalisation, Implications for local decision-makers, Paris: OECD.

PORTER, M.E. (2003): “The Economic Performance of Regions", Regional Studies, 37, 6-7, 549578.

RODRIGUEZ-CASTELLANOS, A.; HAGEMEISTER, M. y RANGUELOV, S. (2010): "Absortptive capacity for R\&D: The identification of different firm profiles". European Planning Studies, Vol. 18, 1267-1283.

TÖDLING, F., PRUD'HOMME VAN REINE, P. y DÖRHÖFER, S. (2011): “Open Innovation and Regional Culture-Findings from Different Industrial and Regional Settings", European Planning Studies, 19:11, 1885-1907.

VON HIPPEL, E. (1988): The sources of innovation. Oxford University Press, New York.

WILLIAMSON, O.E. (1975): Markets and hierarchies: Analysis and antitrust implications. Free Press, New York. 


\section{Gipuzkoaren kanpo harremanak, esku onetan.}

Las relaciones externas de Gipuzkoa, en buenas manos.

Les relations externes de Gipuzkoa, en bonnes mains. 